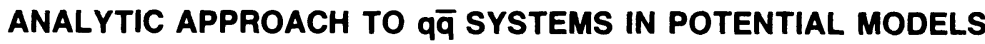

\author{
LALI CHATTERJEE \\ Physics Department \\ Jadavpur University \\ Calcutta - 700032, India \\ and

\section{S. BHATTACHARYYA} \\ Gokhale Memorial College \\ Calcutta - 700020, Ind1a \\ (Received May 7, 1985 and in revised form January 21, 1986)
}

ABSTRACT. Analytic solutions for $\mathrm{q} \overline{\mathrm{q}}$ systems obtained from a cut-off type approximation to the funnel potential are applied to $\bar{b} \bar{b}$ and $\bar{c} \bar{c}$ systems. Perturbative corrections to oscillator energy levels due to inclusion of short range a/r effect are also obtained.

REY WORDS AND PHRASES. Potential Models, Schröodinger Equations. 1980 AMS SUBJECT CLASSIFICATION CODE. 81 .

\section{INTRODUCTION.}

Bound state characteristics of heavy quark-antiquark pairs are generally well described in the framework of non-relativistic potential models. The funnel potential

$$
v_{1}(r)=-a / r+g r+c
$$

which takes both confinement and asymptotic freedom into account by the linear and inyerse $r$ terms respestjuel, $\mathrm{v}_{\mathrm{r}}$ is generally sonsidered the most appropriate [1]. It has been shown $y$ inverse scattering methods [2], [3], using charmonium and upsilon parameters that quarkonium potentials are flavour-independent for the range $0.1 \mathrm{fm}$ < $r<1 \mathrm{fm}$. It has also been found that solutions obtained from $v_{1}(r)$ are generally in qualitative, and often in quantitative agreement with those obtained from the oscillator potential $\mathrm{v}_{2}(\mathrm{r})=\mathrm{Kr}^{2} / 2$.

However, $V_{2}(r)$ completely ignores the short-range Coulombic type potential. As radial wave functions evaluated at the origin are required in expressions for the decay width, it does not seem a good approximation to extrapolate $v_{2}(r)$ to $r=0$ when it is $(1 / r)$ part of $V_{1}(r)$ that dominates close to $r=0$.

In this communication we approximate $v_{1}(r)$ by a cut-off type potential

$$
\begin{aligned}
\mathrm{V}(\mathrm{r}) & =-\mathrm{a} / \mathrm{r} & & 0<\mathrm{r}<\mathrm{r}_{1} \\
& =\mathrm{Kr}^{2} / 2 & & \mathrm{r}_{1}<\mathrm{r}<\mathrm{r}_{2} \\
& =0 & & \mathrm{r}_{2}<\mathrm{r}<\infty
\end{aligned}
$$

with $\mathrm{K}=\mathrm{mw}_{0}^{2} \cdot \mathrm{m}=$ reduced mass of the system. 
We investigate the solution obtained for Quarkonium systems in such a potential, and compare the results with those using $v_{1}(r)$.

The use of $V(r)$ permits us to obtain exact analytic wave functions which are not possible with $V_{1}(r)$, and avoids the necessity of neglecting the Coulombic part of $v_{1}(r)$ that occurs if $v_{2}(r)$ alone is used. We take $a=.27$ from funnel potential parameters [3].

We calculate also the perturbation to the oscillator energy due to the inclusion of $a(-a / r)$ potential for $0<r<r_{1}$, relying heavily on the assumption that the colour interaction has a small coupling constant for small $r$, thereby justifying use of perturbation techniques.

\section{THE WAVE FUNCTIONS POR RACH REGION.}

The radial Schrodinger equation which is operative for a potent1al $(-a / r)$ can be reduced to the confluent form

$$
x \frac{\partial^{2} y}{\partial x^{2}}+(c-x) \frac{\partial y}{\partial x}-a y=0
$$

with

$$
x=\alpha r, c=2 \ell+2, a=\ell+1-\lambda
$$

This yields general solutions $F(a, c, x)$ and $\Psi(a, c, x)$ for integer $c$ which coincides with our case. As $\Psi(a, c, x)$ is not regular at $x=0$ (or $r=0$ ) we reject this, and retain $F(a, c, x)$ as the solution. So,

$$
\mathrm{R}_{1}=A \mathrm{e}^{-\alpha \mathrm{r} / 2} \mathrm{~F}_{1}(\ell+1-\lambda, 2 \ell+2, \alpha \mathrm{r})
$$

where

$$
\alpha=2 \sqrt{2 \mathrm{mE}^{\prime}}, \quad \lambda=a \sqrt{2 \mathrm{~m}} /\left(2 \sqrt{\mathrm{E}^{\prime}}\right), \mathrm{E}^{\prime}=|\mathrm{E}|
$$

We assume $m=$ mass of the constituent quarks of the heavy bound quarkonium system.

The radial schrodinger equation with non-zero $\ell$ for potential $v_{2}(r)=\mathrm{Kr}^{2} / 2$ can be reduced by suitable substitutions to the form

$$
\frac{\partial^{2} y}{\partial z^{2}}+\left\{\left(\ell+\frac{3}{2}\right)-z\right\} \frac{\partial y}{\partial z}-\left(\frac{\lambda^{\prime}+2+3}{4}\right) y=0
$$

where $z=(\alpha r)^{2}$ for the range

$$
r_{1}<r<r_{2}
$$

This yields solutions for $R$ as

$$
\begin{gathered}
\mathrm{R}_{2}=\mathrm{B} \mathrm{e}^{-\left(\alpha^{\prime} \mathrm{r}\right)^{2} / 2}\left(\alpha^{\prime} \mathrm{r}\right)_{1}^{\ell} \mathrm{F}_{1}\left(\frac{\lambda^{\prime}+2 \ell+3}{4}, \ell+3 / 2,\left(\alpha^{\prime} \mathrm{r}\right)^{2}\right) \\
+\mathrm{C} \mathrm{e}^{-\left(\alpha^{\prime} \mathrm{r}\right)^{2} / 2}\left(\alpha^{\prime} \mathrm{r}\right)^{-2 \ell-2} \mathrm{~F}_{1}\left(\frac{\lambda^{\prime}-2 \ell+1}{4}, \frac{1}{2}-\ell,\left(\alpha^{\prime} \mathrm{r}\right)^{2}\right)
\end{gathered}
$$


where

$$
\alpha^{\prime}=4 \sqrt{2 \mathrm{mK}}, \quad \lambda^{\prime}=\mathrm{E}^{\prime} \sqrt{\frac{2 \mathrm{~m}}{\mathrm{~K}}}, \quad \hbar=\mathrm{c}=1 \text {. }
$$

\section{ESTIMATE OF HEAVY QUARK MASSES.}

At the outer boundary, which we expect to be the hadronic radius or confinement radius $\mathrm{r}_{2}$,

$$
\mathrm{R}_{2}=0
$$

and at the inner boundary

$$
\mathrm{R}_{1}=\mathrm{R}_{2}
$$

For values of $E=3100 \mathrm{MeV}$ [4], $\omega_{0}=300 \mathrm{MeV}$ [3] we have evaluated m, using the boundary conditions. We note, for $m=1 \mathrm{GeV}$ and $r_{1}$ and $r_{2}$ are of order of inverse pion mass $\left(M_{\pi}\right) \cdot\left(\alpha^{\prime} r_{1}\right)^{2},>23$ and $\left(\alpha r_{1}\right)>36$.

So that asymptotic expansion of the confluent hypergeometric functions at the boundary may be considered valid. This yields the ratio of the wave function and their derivatives at the boundary, as the equation

$$
\mathrm{H}\left[\frac{\alpha}{2}-(1+\lambda / \alpha r)\right]=\frac{B}{\Gamma\left(\frac{\lambda^{\prime}+3}{4}\right)}\left[\mathrm{H}_{1}+\mathrm{H}_{2}+\mathrm{H}_{3}\right]+\mathrm{H}_{4}
$$

where

$$
\begin{aligned}
\mathrm{H}_{1}= & {\left[-\alpha^{\prime} / 2\left(\alpha^{\prime} \mathrm{r}_{1}\right)^{2}\right], \mathrm{H}_{2}=\left[1 / 2\left(\alpha^{\prime} \mathrm{r}_{1}\right)^{2}\right], } \\
\mathrm{H}_{3}= & {\left[\alpha^{\prime 2} \mathrm{r}_{1}+\left(\lambda^{\prime} / 2-1 / 2\right) /\left(\alpha^{\prime} \mathrm{r}_{1}\right)\right] /\left[2\left(\alpha^{\prime} \mathrm{r}_{1}\right)\right] } \\
\mathrm{H}_{4}= & \mathrm{C}\left[\left(\alpha^{\prime 2} \mathrm{r}_{1}\right)+\left(\lambda^{\prime} / 2-1 / 2\right) /\left(\alpha^{\prime} \mathrm{r}_{1}\right)\right] / \Gamma\left(\frac{\lambda^{\prime}+1}{4}\right) \\
& ((\lambda+1) / \alpha \mathrm{r})<\alpha / 2 \text { and }\left[\left(\lambda^{\prime} / 2-1 / 2\right) /\left(\alpha^{\prime} \mathrm{r}_{1}\right)\right]<\alpha^{\prime 2} \mathrm{r}_{1} \\
\mathrm{H}= & {\left[\mathrm{B} /\left\{2 \alpha^{\prime} \mathrm{r}_{1} \Gamma\left(\frac{\lambda^{\prime}+3}{4}\right)\right\}\right]+\left[\mathrm{C} / \Gamma\left[\left(\frac{\lambda+1}{4}\right)\right]\right.}
\end{aligned}
$$

From (3.2) we have $B=p c$, where

$$
\begin{aligned}
& \mathrm{p}=-\frac{1}{\alpha^{\prime 2} \mathrm{r}_{2}} \frac{\left(\frac{\lambda^{\prime}+3}{4}\right) \Gamma(1 / 2)\left(\alpha^{\prime} \mathrm{r}_{2}\right)^{1 / 2}}{(1 / 2) \Gamma\left(\frac{\lambda^{\prime}+1}{4}\right) \Gamma(1 / 2)} \\
& \mathrm{p}=-.06 \text { for } \mathrm{m} \approx 1 \mathrm{GeV} .
\end{aligned}
$$

Equation (3.3) can be reduced to

$$
\sqrt{2 \mathrm{mE}^{\prime}}=\frac{p 4 \sqrt{2} \sqrt{\mathrm{m} \omega_{0}}}{2} \frac{\Gamma\left(\frac{\lambda^{\prime}+1}{4}\right)}{\Gamma\left(\frac{\lambda^{\prime}+3}{4}\right)}+r_{1} \sqrt{2} \mathrm{m \omega}_{0}
$$


Neglecting the lst term on the r.h.s., since it is of order $.02 \mathrm{~m}$, compared to $\sim 1.5 \mathrm{~m}^{3}$ and $87 \mathrm{~m}^{2} \sqrt{\mathrm{m}},(\mathrm{m} \sim 1 \mathrm{GeV})$, we estimate

$$
\sqrt{\mathrm{m}}=\overline{\sqrt{E^{\prime}} / \mathrm{r}_{1} \omega_{0}}
$$

Thus $m$ is sensitive to the value of $r_{1}$ and $\omega_{0}$, and is not dependent much on $r_{2}$ and on the magnitude of $a$, when a is small.

We find the funnel potential prediction of mass $m=1650 \mathrm{MeV}$ is obtained for $r_{1}=.0046 \mathrm{MeV}^{-1}$ when $\omega_{0}=300 \mathrm{MeV}$.

We can then choose $\underline{r}_{\underline{2}_{1}}=1 / M_{\pi}=.0073 \mathrm{MeV}^{-1}$. Alternatively, if we wish to $\mathrm{f}_{1 x} \mathrm{r}_{1}$ at $1 / 2$ fermi $=.0025 \mathrm{MeV}^{-1}$, which is assumed generally to be the region where inverse $r$ behaviour falls of $f$ and the linear confining effect sets in, we get a much larger value for $m=5.5116 \mathrm{GeV}$.

Further, from $\left|R_{1 s}(r=0)\right|^{2}=0.5(\mathrm{GeV})^{2}$ we can get straight away $A=\sqrt{5 \times 10^{6}}=$ 707.107 from Eq. (2.2). Similarly $B$ and $C$ may be found.

If we assume the Upsilon $V$ potential to be of the same form with the same value of $\omega_{0}$ as obtained by level fitting from charmonium a value of $\sim 5 \mathrm{GeV}$ for the mass of the beauty quark is obtained.

A value $372 \mathrm{MeV}$ for $\omega_{0}$ obtained by level fitting of $1 \mathrm{~s}, 2 \mathrm{~s}$ levels of $V$ yields a lower value of $\mathrm{m}_{\mathrm{b}}$.

For the case of Upsilon, using $E=9460$ [4], we obtain by simflar analysis $\mathrm{m}=$ 4967.416 for the beauty quark with Coulombic radius $=0.0046 \mathrm{MeV}^{-1}$ while a value 372 $\mathrm{MeV}$ for $\omega_{0}$ from $v$ level fitting yields $\mathrm{m}_{\mathrm{q}}=3230.647 \mathrm{MeV}$ for $\mathrm{r}_{1}=0.0046 \mathrm{MeV}^{-1}$.

The analytic solutions obtained can be used in problems involving the nonrelativistic potential models for heavy quarkonium systems assuming the potential to be flavour independent.

\section{CORRECTIONS TO ENERGY LEVELS.}

We now estimate the correction to the first oscillator energy level due to the perturbation by the Coulombic potential for $r$ between zero and $r_{1}$.

The perturbation energy for the $\mathrm{L}=0, \mathrm{~J}=0$ level may be written

$$
\Delta \mathrm{E}=\int\left(\mathrm{V}-\mathrm{V}_{0}\right) \Psi_{0} \mathrm{dr}
$$

where $\Psi_{0}=$ the unperturbed wave function and the perturbation potential $\left(V-V_{0}\right)$ is

$$
\begin{array}{rlrlrl}
\left(\mathrm{V}-\mathrm{V}_{0}\right) & =\mathrm{a} / \mathrm{r}-\mathrm{V}_{0} & & \text { for } & 0<\mathrm{r}<\mathrm{r}_{1} \\
& =0 & & \text { for } & \mathrm{r}_{1}<\mathrm{r}<\mathrm{r}_{2} \\
& =-\mathrm{v}_{0} & \text { for } & \mathrm{r}_{2}<\mathrm{r}<\alpha
\end{array}
$$


where $\mathrm{V}_{0}=$ unperturbed potential.

Due to confinement $\Psi_{0}=0$ for $r>r_{2}$, and we have eventually

$$
\Delta E=4 \pi\left(\alpha^{\prime} / 2 \pi V\right)\left[-a I_{1}-K_{2} / 2\right]
$$

where $I_{1}=\int_{0}^{r_{1}} r e^{-b r^{2}} d r$ and $I_{2}=\int_{0}^{r_{1}} r^{4} e^{-b r^{2}} d r$ we have used for the unperturbed

$$
\Psi_{n}=\left(\frac{\alpha}{\sqrt{2} 2^{n} n !}\right)^{1 / 2} e^{-(\alpha r / 2)^{2}} H_{n}(\alpha r)
$$

as given by [6].

$I_{1}$ and $I_{2}$ are evaluated using the error function [6]. We get, for $r_{1}=-0026 \sim$ $.5 \mathrm{fm}, \omega_{0}=300 \mathrm{MeV}, \mathrm{m}_{\mathrm{c}}=1.17 \mathrm{GeV}$.

$$
\Delta \mathrm{E}=-0.002 \mathrm{MeV}
$$

Alternatively if we take $r_{1}=.0020, \mathrm{~m}_{\mathrm{q}}=1.65 \mathrm{MeV}$, retaining $\omega_{0}=300 \mathrm{MeV}$, we obtain

$$
\Delta \mathrm{E}=-0.0035 \mathrm{MeV}
$$

The small magnitude of the perturbation energy shows that perturbation corrections are justified. However, if the short range coupling was greater than $a=.27$, the perturbation energy would increase correspondingly through $\left(\mathrm{aI}_{1}\right)$ term.

ACRNowl EDGEMENT: The first author wishes to thank U.G.C., India for financlal support for the research.

\section{REPERBNCES}

1. NOVIKOV, V.A., et. al. Charmonium and Gluons, Physics Reports 41C 1978, 1-135.

2. QUIGG, C. and ROSNER, J. L. Further Evidence for Flavour Independence of the Quark-antiquark Potential, Phys. Rev. D23 1981, 2625-2637.

3. MoXHAY, P., ROSNER, J. L. and QUIGG, C. Beyond $\underline{Y}$ : Heavier Quarkonfa and Interquark Force, Phys. Rev. D23 1981, 2638-2646.

4. ERDELEI, A. (Editor) Higher Transcendental Functions I., MaGraw Hill Book Co. 1953.

5. FLUGGE, S. Practical Quantum Mechanics, Springer Verlag 1, 1974.

6. ABRAMOVITCH, M. and STEGON, I. A. Hand Book of Mathematical Functions, Dover PubPublications Inc., NY, 1965. 


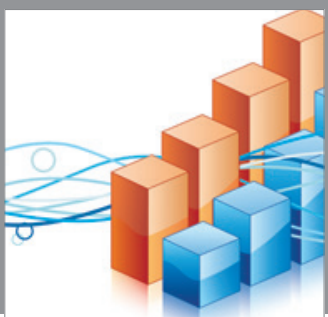

Advances in

Operations Research

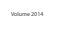

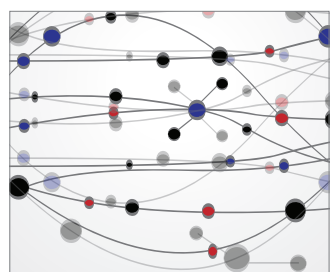

\section{The Scientific} World Journal
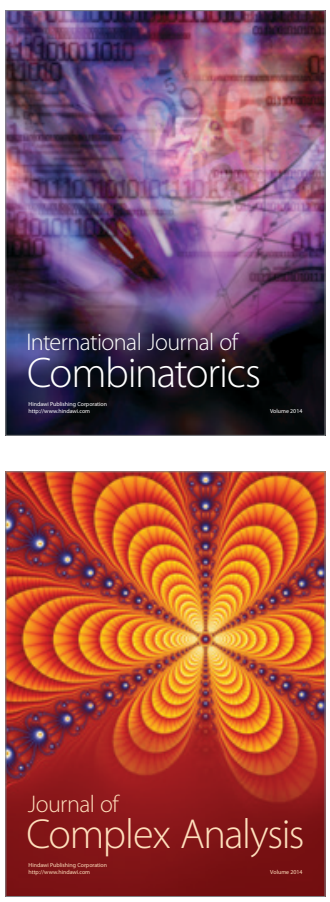

International Journal of

Mathematics and

Mathematical

Sciences
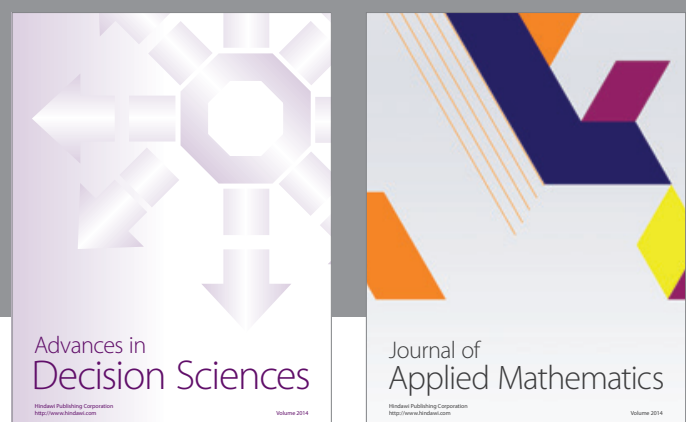

Journal of

Applied Mathematics
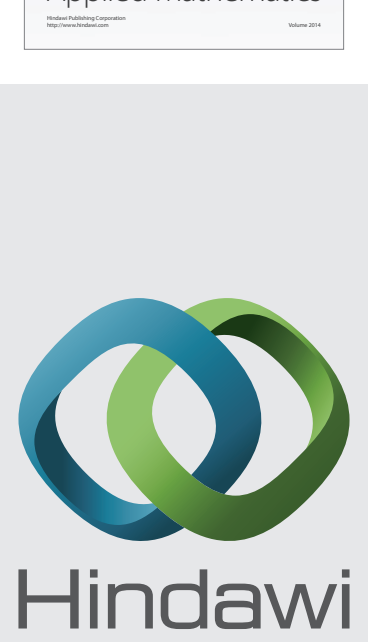

Submit your manuscripts at http://www.hindawi.com
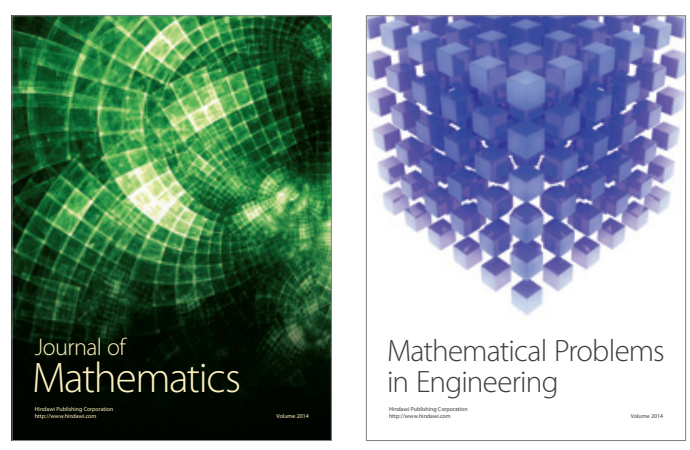

Mathematical Problems in Engineering
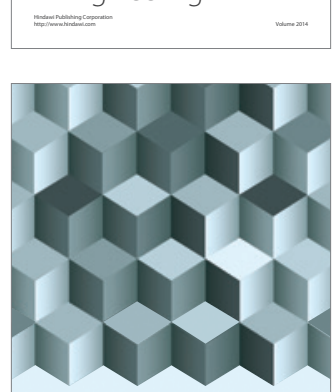

Journal of

Function Spaces
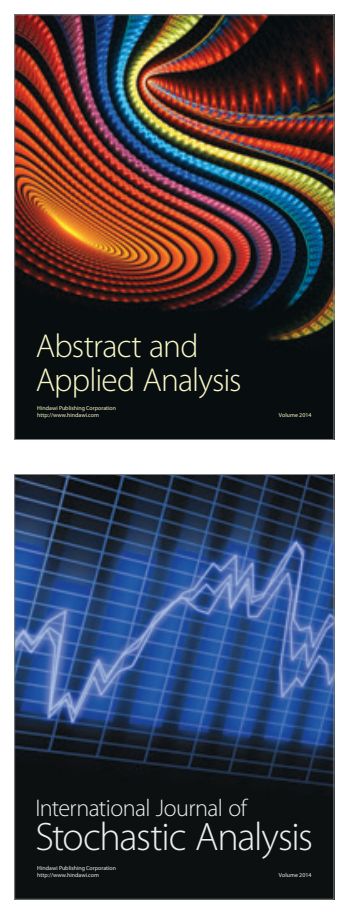

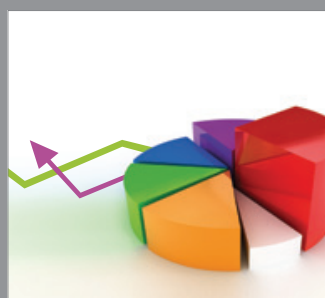

ournal of

Probability and Statistics

Promensencen
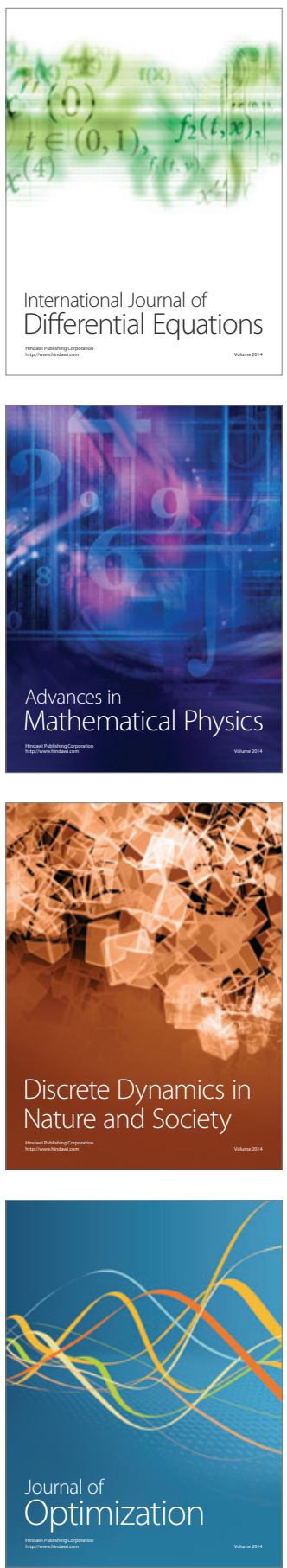